(c) 2010 IEEE. Personal use of this material is permitted. Permission from IEEE must be obtained for all other uses, in any current or future media, including reprinting/republishing this material for advertising or promotional purposes, creating new collective works, for resale or redistribution to servers or lists, or reuse of any copyrighted component of this work in other works. 


\section{Torpid Mode: Hybrid of Sleep and Idle Mode as Power Saving Mechanism for IEEE 802.16j}

\author{
Ashish Kumar Singh \\ Wireless Protocols and Platforms Group, \\ Samsung India Software Operations, \\ Bangalore, India. \\ ashish.ism.cse@gmail.com
}

\author{
Vidyasagar Potdar \\ Digital Ecosystems and Business Intelligence \\ Institute, Curtin University, \\ Perth, Australia. \\ vidyasagar.potdar@cbs.curtin.edu.au
}

\begin{abstract}
Sleep and Idle modes are proposed as power saving mechanisms in IEEE 802.16j Mobile Multi-hop Relay (MMR) networks. In this paper, these mechanisms are analyzed in terms of number of messages exchanged, latency and cost. Besides, hybridization of Sleep and Idle modes, named as Torpid mode is proposed as a power saving mechanism for IEEE 802.16j. Our proposed mechanism takes advantage of the relay stations. Analytical results verified by simulations evince that our proposed mechanism surpasses both Idle and Sleep modes significantly in terms of bandwidth consumption. Torpid mode also outperforms over the Idle mode in terms of latency and is superior to Sleep mode on the grounds of cost.
\end{abstract}

Keywords-IEEE 802.16j; power saving modes; Sleep mode; Idle mode

\section{INTRODUCTION}

In an attempt to deliver high data transmission rates, a new WiMAX working group was established, named as IEEE 802.16's Relay Task Group. The group came up with development of a new standard, which is commonly known as Mobile Multi-hop Relay (MMR) [1]. Assuming that Relay Stations (RSs) can be developed at cost significantly less than that of Base Stations (BSs), this standard primarily aims at providing low cost coverage in initial stages of network deployment and secondly to provide increased capacity in case of higher utilization of the network [2]. Furthermore, this standard reduces power consumption of Mobile Station (MS) as MS is connected to the RS rather than relatively far BS [3].

MSs in the mobile systems based on MMR standard are powered by battery. As batteries have limited power to supply, it is always desired to minimize power consumption. Hence, power efficient operation of MS is one of the factors of prime importance that will affect the wide deployment of the Mobile WiMAX [4].

Rest of the paper is organized as follows. The related work is briefed in Section II. Our proposed Torpid mode is described in Section III. In Section IV, details of our evaluation methodologies are described.
Performance analysis of our proposed model is made in Section V. Concluding remarks are made in Section VI of the paper.

\section{RELATED WORK}

One approach to achieve power efficient operation of MS is to allow MS to power down operations related to communication with a BS or RS for periods of inactivity. Two mechanisms that follow this approach, viz., Sleep mode and Idle mode are specified in IEEE 802.16 e. Recently some suggestions have been made for handling of IEEE 802.16e messages and inclusion of new parameters to assist the Sleep and Idle mode operations in IEEE 802.16j [5].

High power saving efficiency and low latency of returning to Awake mode are desired features of any power saving mechanism. Though Sleep and Idle modes can be used in IEEE $802.16 \mathrm{j}$, they improve only one of these features at other's cost and are better suited to different scenarios. Moreover, they do not completely utilize the presence of RSs. These inadequacies of existing mechanisms and the need of power efficient operation of MSs motivated us to devise a mechanism that incorporates the advantages of existing mechanisms and reduces the exchange of control messages to trim down bandwidth consumption. In the next section we elucidate how our proposed mechanism achieves this goal.

\section{THE TORPID MODE}

In this section, we briefly describe the concept and operation of the Torpid mode.

\section{A. Concept}

The concept of Torpid mode lies with the fact that Sleep mode is profitable for MSs which do not frequently move from one cell (union of coverage areas of current serving BS and RSs in its coverage area) to another cell. On the other hand, Idle mode is advantageous if MSs are highly mobile. Also, presence of RSs in IEEE $802.16 \mathrm{j}$ provides an opportunity to decrease exchange of control packets and latency. 
It is highly probable that a MS with short period of inactivity might awake in the same cell. On the other hand, a MS with long period of inactivity is likely to move out of the cell before termination of its inactivity period. Because of above mentioned reasons, it would be prudent to use Sleep mode for small inactivity periods and Idle mode for large inactivity periods.

\section{B. Basic Operation}

Basic operation of Torpid mode can be divided into two parts, viz. T-Sleep mode operation and T-Idle mode operation, as shown in Fig. 1. Td is the time of inactivity required to trigger the mechanism. During TSleep mode, the MS sleeps and remains in Idle mode during T-Idle mode. Ts is the time after which a MS in T-Sleep mode switches to T-Idle mode. It can be determined by either of the BS, RS and MS or by the collaboration of all. It should be noted that MS with inactivity period smaller than Ts never switches to Idle mode.

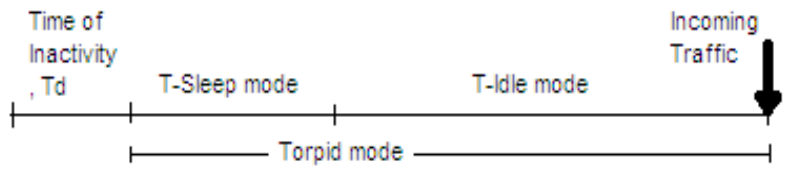

Fig 1. Dissection of Torpid mode into T-Sleep and T-Idle modes

\section{TABLE I: TORPID MODE ALGORITHM}

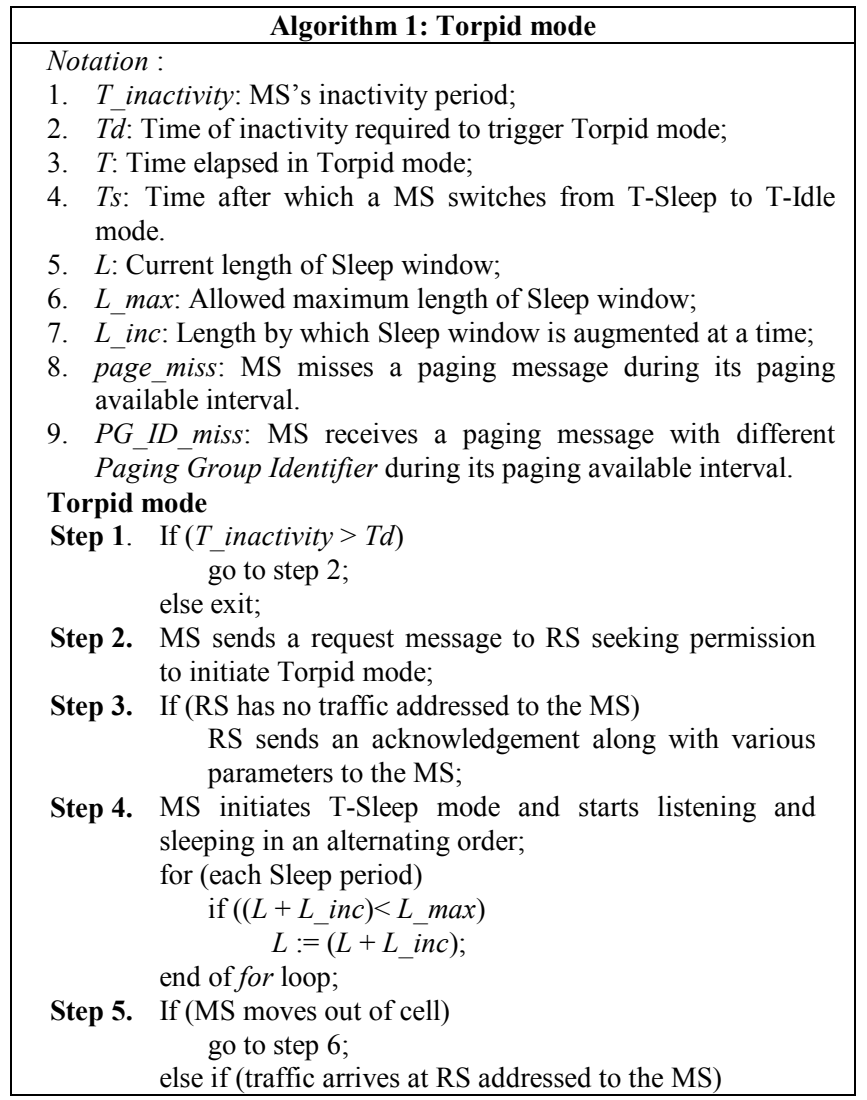

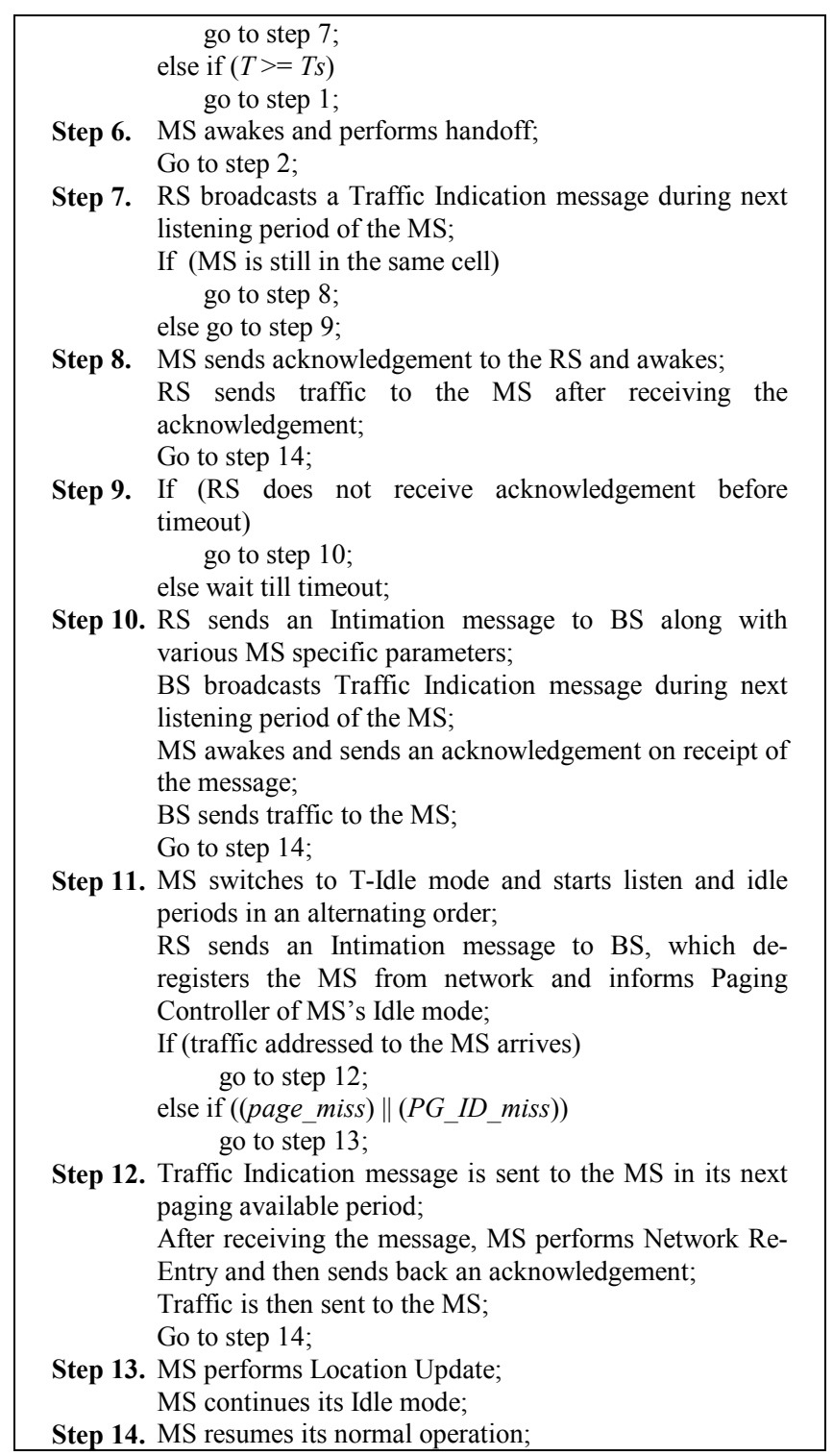

The algorithm given in Table I, shows the flow of operation involved during Torpid mode. After Td time of inactivity an MS requests RS to allow it to initiate Torpid mode. RS processes this request and sends back an acknowledgement along with various parameters like, Ts, Sleep window length, listen window length, paging available period, paging unavailable period, paging group identifier, etc. On receipt of the response and various parameters, MS initiates T-Sleep mode. During T-Sleep mode, MS sleeps and listens in an alternating order. Depending upon type of service, length of Sleep window can be fixed or can increase with time, till it attains maximum possible value. For services with a constant transmission rate such as Voice over IP (VoIP), it is necessary that Sleep window sustains a constant length. T-Sleep mode is terminated in following three conditions. 
1) Positive Traffic Indication message from RS: When RS receives some traffic before Ts time, it sends a Traffic Indication message (MOB_TRF-IND) to the MS in Sleep mode to which traffic is addressed. To make sure that MS receives the message, RS keeps record of transmission delay, and listening and sleeping intervals of each MS in Sleep mode. With help of these records, RS sends the Indication message during next listening interval of the MS. On receipt of the message, MS terminates the Torpid mode and acknowledges RS by sending an acknowledgement (MOB_AWK-ACK). However, it is possible that MS might move out of RS's coverage area during T-Sleep mode, before RS sends Traffic Indication message to it. The Indication message thus misses the MS. This case is referred as Slip Case. As MS receives no Indication message, it sends no acknowledgement to the RS. After sending the Indication message to MS, RS waits for some time (inclusive of RS to MS and MS to RS transmission delays). If no acknowledgement is received by the RS in this time, it sends an Intimation message (MOB_SLPINT) along with the traffic to BS. However, the need to send back the traffic to BS can be avoided by caching all incoming traffic for Ts time at BS. BS then broadcasts Traffic Indication message. On receipt of Indication message, MS awakes and sends back an acknowledgement. BS then sends the traffic to MS.

2) Handoff: If MS, currently in T-Sleep mode, moves away from the current cell (serving cell), then it turns back to Awake mode and handle handoff as it would have handled during its normal operation. After performing handoff, it can again initiate Torpid mode. However, the need of terminating T-Sleep mode to perform handoff pulls down the power saving efficiency of the mechanism.

3) Completion of T-Sleep mode time, (T>TS): A MS switches to Idle mode after spending Ts time in T-Sleep mode. It does not need to turn into Awake mode for doing so as all the parameters needed for Idle mode operations are acquired by it at the beginning of the Torpid mode. At the time when MS switches to Idle mode, RS intimates BS to deregister MS from the network and inform PC about MS's Idle mode. MS remains de-registered through out its T-Idle mode. Following sub-sections elaborate on T-Idle mode operation.

\section{Paging Group}

Functioning of T-Idle mode is based on Paging Groups (PG). The concept of PG is to cluster some BSs, along with their respective associated RSs, together in a way that seamless movement of a MS in Idle mode is allowed within a cluster. Every single cluster is known as a PG. Each PG has a Paging controller (PC). PC is responsible for assigning Paging group identifier. Each member BS and RS of PG is set to have the same configuration, including Paging group identifier. List of MSs in Idle mode are shared by all the members of a PG and so location of the MSs within a PG is not a problem. Fig. 2 shows how cells are clustered to form PGs. Cells with same numbers belong to same PG.

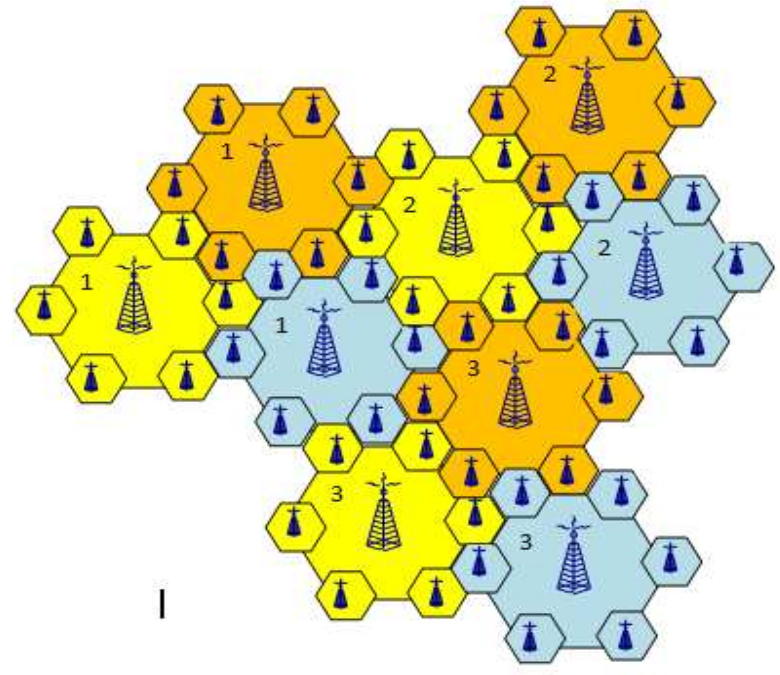

Fig 2. Clustering cells into paging groups

In T-Idle mode MS listens and remains idle in an alternating order for paging available period and paging unavailable period respectively. MSs in T-Idle mode are paged during each of their paging available intervals. Paging message, MOB_PAG-ADV, includes Paging group identifier and serves two purposes. Firstly, paging helps a MS in T-Idle mode to decide whether it is in the same PG or has moved to some other PG. Secondly, in case of arrival of some traffic addressed to the MS, paging intimates the MS in the very next paging interval to terminate its Torpid mode.

\section{Location Update}

If a MS, currently in T-Idle mode, moves away from its current PG then it must perform Location Update (LU). The need of LU is determined by the MS based on the fact that each PG has a unique Paging group identifier and other PGs have no idea about the paging available intervals of the MS. Therefore, if the MS misses a paging message in paging available interval or receives a paging message with different Paging group identifier then it performs Location Update. During Location update, the MS updates Paging group identifier, and paging available and unavailable periods.

\section{E. Network Re-entry}

On arrival of traffic, addressed to a MS in Idle mode, the MS is paged informing this in its next paging available interval. MS then terminates T-Idle mode and obtains, negotiates, adjusts, and updates the cell- 
specific parameters. This process is known as Network Re-Entry and is unavoidable in case of T-Idle mode because MS remains de-registered throughout the mode. It increases the overhead associated with Idle mode.

\section{EVALUATION METHODOLOGY}

In this section we present the grounds on which we evaluated and compared different modes. We carried out comparisons on following three grounds.

\section{A.. Latency}

Latency is a measure of delay in delivery of incoming traffic and so is an undesired overhead incurred in all power saving mechanisms. For each mode we define different latencies, viz., Sleep latency, Idle latency and Torpid latency. Efficiency of any power saving mechanism depends on the length of Sleep window or Idle window (paging unavailable period). The more is the length of the window, the more is the efficiency of the mechanism. However, more length of the window implies more latency. For that reason, efficiency and latency are inversely proportional to each other.

\section{B. Message exchanging}

Messages exchanged between MS, RS and BS consumes bandwidth and so it is desirable to minimize it. In 802.16j MMR networks, it is not only the MSs that communicate with the BS but also the RSs. Therefore, saving bandwidth holds utmost importance in 802.16j MMR networks. Reduced control message exchange diminishes the bandwidth consumption, which as a result lessens the overall call incompletion probability.

For capturing exchange of messages during simulations, some assumptions are made. We assume that packets are large enough to carry any message. Also, if a message reaches MS from BS via RS, then that one message is counted as two.

\section{Cost}

Based on the evaluation methodology used in [6], we have derived similar cost equation for our mechanism. In this evaluation methodology power consumed during different modes is calculated taking into account power consumed during message transactions for handover, location update, or network re-entry and the power consumed while decoding downlink frames in listening or paging intervals. The cost equations we have used for different modes are as follows:

$$
\mathrm{C}_{s}=\mathrm{C}_{l}+\mathrm{C}_{h}
$$

$$
\begin{aligned}
\mathrm{C}_{i} & =\mathrm{C}_{p}+\mathrm{C}_{u}+\mathrm{C}_{e} \\
\mathrm{C}_{t} & =\mathrm{C}_{s}+\mathrm{C}_{i} \\
& =\left(\mathrm{C}_{l}+\mathrm{C}_{p}\right)+\left(\mathrm{C}_{h}+\mathrm{C}_{u}\right)+\mathrm{C}_{e}
\end{aligned}
$$

$\mathrm{C}_{s}$ is cost for Sleep mode.

$\mathrm{C}_{i}$ is cost for Idle mode.

$\mathrm{C}_{d}$ is cost for Torpid mode.

$\mathrm{C}_{l}$ is cost for decoding frames in listening period.

$\mathrm{C}_{p}$ is cost for decoding frames in paging period.

$\mathrm{C}_{h}$ is cost for handoff.

$\mathrm{C}_{u}$ is cost for location update.

$\mathrm{C}_{e}$ is cost for network re-entry.

For calculation of cost equations, a unit value is assigned to $\mathrm{Cl}$ and $\mathrm{Cp}$. The ratio between the power consumed by uplink transmission and downlink decoding is assumed to be 1 [6]. Usual four message transactions are considered for ranging, capability negotiation, privacy key exchange and registration [6]. Consequently a handoff or network re-entry process is assumed to have a cost that is eight times of the unit cost. We have only considered unsecure location update that has a cost same as handover or network re-entry. For convenience, unit cost is set to 1 . Therefore, $\mathrm{Ch}$ : $\mathrm{Cu}: \mathrm{Ce}: \mathrm{Cl}: \mathrm{Cp}=8: 8: 8: 1: 1$.

\section{PERFORMNCE ANALYSIS}

In this section, performance analysis of our proposed Torpid mode is made by using $\mathrm{C}++$ programming. The simulation setups and results are described as follows.

\section{A. Simulation Setups}

We have simulated our proposed model for detailed operations of Sleep, Idle and Torpid modes in IEEE $802.16 \mathrm{j}$ using $\mathrm{C}++$ programming. In our model, we refer either of Sleep, Idle and Torpid modes as an event. Event arrival rate is made to follow an exponential distribution with mean defined as inverse of Event Arrival Rate (EArrRate). Duration of event is generated for each event and follows an exponential distribution with a mean defined as EventTime. Mobility of MSs is incorporated in the model by generating a residence time for each event. Residence time is the maximum time for which a MS can reside in a cell before either moving out of cell or awaking. Residence time of any event is again a random number that follows an exponential distribution with a mean defined as ResidenceMean.

In the simulations, latency, number of the messages exchanged, handoffs, location updates, network reentries, listening periods and paging periods are recorded. Results obtained from the simulations are then used for calculating cost, total latency and number of messages exchanged. To make the results more precise they are averaged over one million simulations. Based on the discussions in WiMAX Mobile Task 
Group (MTG), values of various parameters required in the simulation are determined [6] and are given in Table II. Length of a frame is assumed to be $5 \mathrm{msec}$ for carrying out calculations in unit of time [7] [8].

TABLE II: LIST OF SIMULATION PARAMETERS

\begin{tabular}{|l|l|l|}
\hline Attribute & Parameter & Value \\
\hline Minimum Sleep window & ts & 2 frames \\
\hline Listening window & tl & 2 frames \\
\hline Maximum Sleep window & tsm & 1024 frames \\
\hline Paging available period & tp & 2 frames \\
\hline $\begin{array}{l}\text { Paging unavailable } \\
\text { period }\end{array}$ & tu & 1024 frames \\
\hline
\end{tabular}

\section{B. Results and Discussion}

In Fig. 3, total number of messages exchanged for varying Ts is compared between different modes. Sleep and Idle modes maintains a zero slope as Ts has got no role to play in these mechanisms. At $\mathrm{Ts}=0$, Torpid mode has no allowed time for T-Sleep mode and so is almost equivalent to Idle mode. As Ts increases number of messages exchanged drops further for Torpid mode. This drop can be credited to the increasing duration of T-Sleep mode as during this mode, unlike in Sleep mode, BS and RS do not need to send no-traffic messages, i.e. MOB_TRF-IND (negative), to MSs in their listening periods.

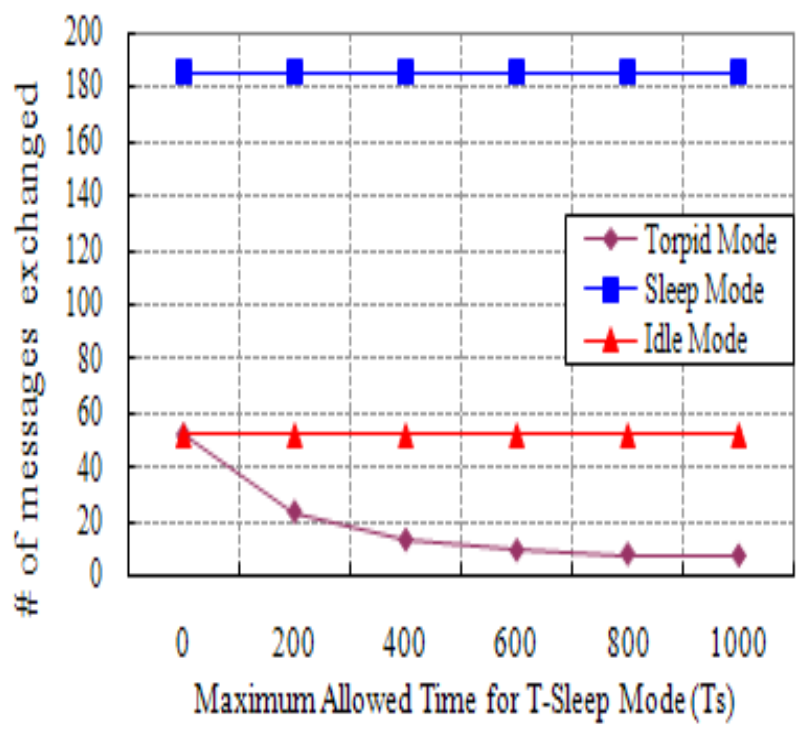

Fig 3. Number of messages exchanged vs. Ts

To understand the effect of mobility of MSs on different power saving mechanisms, different modes are compared for varying values of average residence time, i.e. ResidenceMean. Fig. 4 shows that number of messages exchanged declines, at a declining rate, with increasing ResidenceMean for all the modes. The reason is that Handoff and LU decreases with increasing ResidenceMean.

Cost for each mode is plotted against Ts in Fig. 5. Idle mode's cost remains lowest among all as there are no handoffs in the mode and cost of decoding downlink frames is less. With increasing Ts, number of handoffs and listening periods increases, while that of location updates and paging periods decreases. But the increase in former is much significant and so the cost of Torpid mode increases and becomes constant after Ts grow large enough to turn Torpid mode completely into TSleep mode.

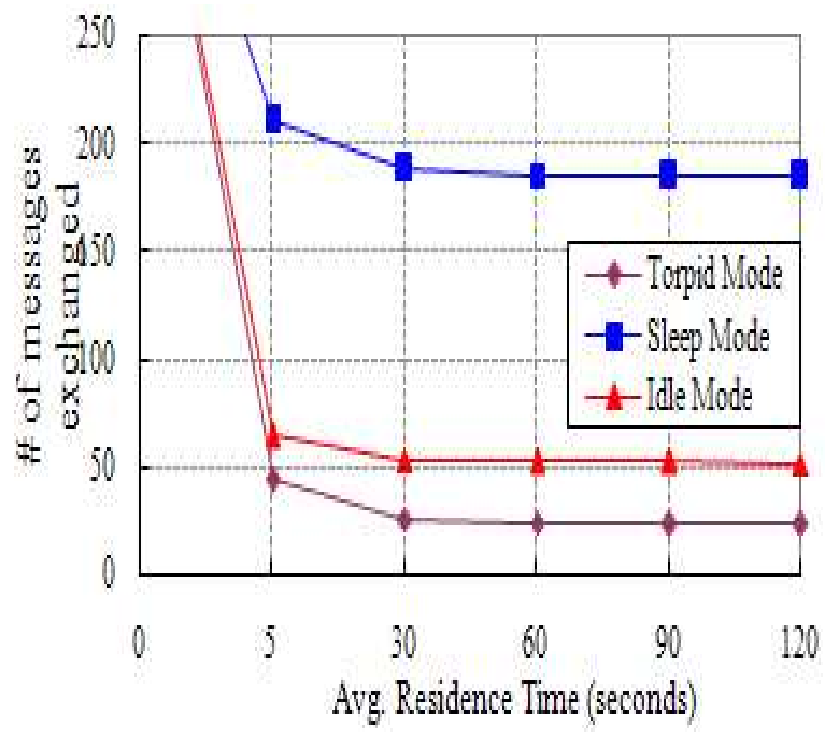

Fig 4. Number of messages exchanged vs. ResidenceMean

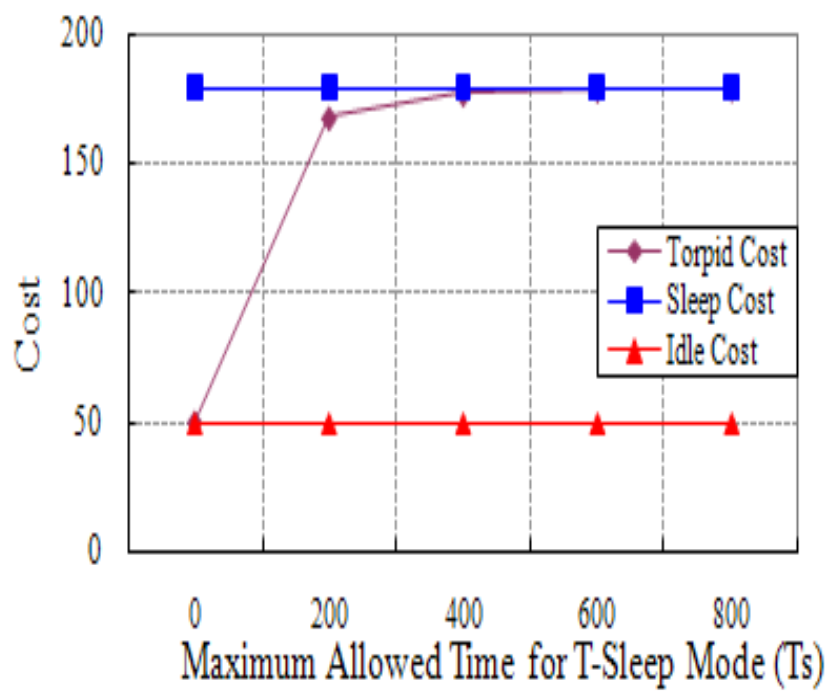

Fig 5. Cost vs. Ts for different modes

Fig. 6 pictures how cost of different modes vary as ResidenceMean increases. Mobility of MSs decreases with increase in ResidenceMean and so the number of handoffs and location updates plummets. Decrease in 
handoffs and LUs pulls down the cost of mechanisms. Therefore, cost decreases for all of the modes with rising ResidenceMean. Though rate of decrease of cost decreases for all the modes, it is highest for Sleep mode, followed by Torpid and Idle modes. Idle mode maintains the lowest cost throughout because, as stated before, there are no handoffs in the mode and the cost of decoding downlink frames is less.

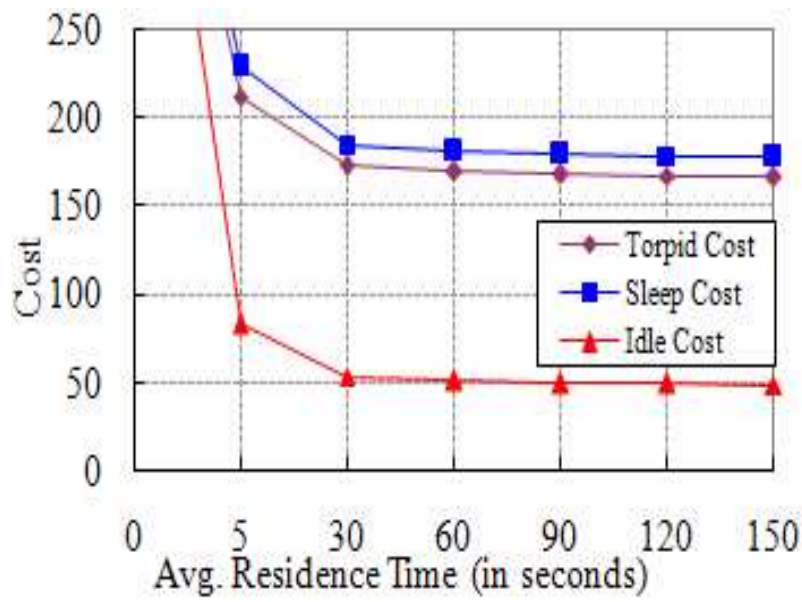

Fig 6. Cost for different modes vs. ResidenceMean

Latency is directly proportional to length of windows in any mechanism and this is demonstrated in Fig. 7. Torpid latency decreases with increasing Ts as for Ts time T-Idle windows are replaced by T-Sleep windows, which are relatively much smaller.

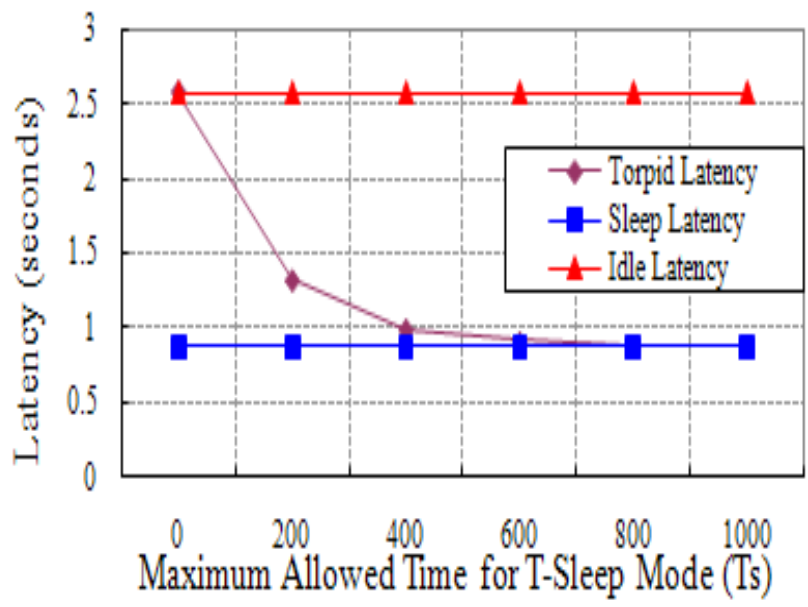

Fig. 7: Latency vs. Ts for different modes.

ResidenceMean has no effect on latency as it does not concern length of windows. Therefore latencies of all the modes remain constant for varying values of ResidenceMean.

\section{CONCLUSIONS}

In this paper, we have analyzed different power saving mechanisms for IEEE $802.16 \mathrm{j}$ on three different parameters, viz., number of messages exchanged, latency and cost. Two of the analyzed mechanisms are the modified versions of Sleep and Idle modes specified in IEEE 802.16e standard, which do not take advantage of the presence of Relay Stations. Based on our study, hybrid of these modes, Torpid mode, has been proposed. As shown in the results, our proposed mechanism outperforms both Sleep and Idle modes. Despite of lowest bandwidth consumption, Torpid mode has a cost much less than that of Sleep mode and a much lower latency as compared to the Idle mode. Furthermore, performance of Torpid mode can be improved significantly by choosing an appropriate value of Ts.

\section{REFERENCES}

[1] R. Marks, et al, "Standards from IEEE802 unleashed the Wireless Internet”, IEEE Microwave Magazine, Volume 2, Issue 2, pp.46-56, June 2001.

[2] Y. Yu, S. Murphy, and L. Murphy, "Planning Base Station and Relay Station Locations in IEEE 802.16j Multi-hop Relay Networks", IEEE CCNC 2008, NV USA, January 2007, pp. 922-926.

[3] S. Ann, K. G. Lee and H. S. Kim, "A Path Selection Method in IEEE 802.16j Mobile Multi-hop Relay Networks", SENSORCOMM'08, NV USA, Aug. 2008, pp. 808-812.

[4] IEEE 802.16-2004, "IEEE standard for local and Metropolitan Area Network-part 16: Air Interface for Fixed Broadband Wireless Access Systems," Oct. 2004.

[5] S. L. Tsao, et al, "Sleep Mode and Idle Mode Operations for IEEE 802.16j", Call for Techinical Proposals regarding IEEE project P802.16j, TX USA, Nov. 2006.

[6] B. Kim, J. Park, and Y. H. Choi, "Power Saving Mechanism of IEEE 802.16e: Sleep Mode vs. Idle Mode", ISPA Ws, Italy, Dec. 2006, pp. 332-340.

[7] The WiMAX Forum: available at http://www.wimaxforum.org/.

[8] A. Talevski, and V. Potdar, "Secure and Mobile Multimedia Convergence", ICHIT, Korea, 2009. 